\title{
Patient's perceptions of medication counselling from community pharmacies
}

\begin{abstract}
Introduction: Through medication counseling interventions, pharmacists can increase patients' knowledge and understanding of their medications, and enable them to make appropriate decisions regarding their therapy. This study explored the perceptions of patients suffering of long term conditions (LTC) on medication counseling received from community pharmacies.
\end{abstract}

Method: This was a cross sectional survey targeted at patients with LTCs who are on regular medications. The survey was conducted in North and South London over a period of 6 months. The study was approved by the academic institute ethics committee.

Results: The survey was completed by 486 patients. Only $48 \%$ received medication counseling, mainly through the medicine use review and new medicine advanced services. Nearly all patients received information regarding the purpose and dosing regimen of the medicine. However, 50\% received information about side effects with only $27 \%$ receiving information on life style changes. Most patients $(80 \%)$ received the counseling information verbally. On a scale of 1 to 5 with 1 not being at all satisfied and 5 extremely satisfied, the majority of patients rated their satisfaction as 3 (average) with the counseling received mostly due to lack of detailed information. Patients wanted to receive information on side effects and life style and receive the information orally and in writing. More than half of the patients $(60 \%)$ read the PIL and found it a useful source. Nevertheless, despite the patient information leaflet (PIL), very few patients recognised receiving written information when being counselled.

Conclusion: Advanced pharmacy services are becoming the forum to offer medication counselling. Counselling content and format need to be improved to meet patients' needs. Although the PIL is being provided with every dispensed medicine and more than half of patients read it, it was not recognised by patients as a written source of medication counseling. This emphasises the need to highlight the PIL as a source of written information when offering medication counselling.
Volume 4 Issue 2 - 2016

Reem Kayyali, Ana Marques Gomes, Thuy

Mason, Minal Naik

School of Pharmacy and Chemistry, Kingston University, United Kingdom

Correspondence: Reem Kayyali, School of Pharmacy and Chemistry, Kingston University, Kingston upon Thames KTI 2EE, United Kingdom, Tel 0208417 256I,

Email R.Kayyali@kingston.ac.uk

Received: November 03, 2014 | Published: April 07, 2016

\section{Introduction}

Over 15million people in the United Kingdom are living with at least one long term condition (LTC) including hypertension, diabetes, chronic obstructive pulmonary disorder, chronic heart disease etc. ${ }^{1}$ Increased incidence of LTC presents a huge challenge to the National Health Service (NHS). ${ }^{2}$ Medications are the main intervention provided for LTC management. However, about $30-50 \%$ of medicines prescribed to patients with LTCs are not taken as recommended by the healthcare professional (HCP). ${ }^{3}$ This results in lack of improvement and deterioration of health, increase in demand for healthcare and wastage of medicines. ${ }^{3}$ A Cochrane review concluded that interventions to improve medication taking as opposed to treatment of LTCs may reduce non-adherence and provide a far greater impact on clinical outcomes. ${ }^{4}$

Several authors have researched different intervention strategies to improve medication adherence in patients with LTC and their effects, however, no single intervention strategy can improve adherence of all patients, rather, a combination of approaches appeared to be best. ${ }^{5,6}$ Intervention strategies that have been researched are mostly grouped into: behavioral interventions, designed to change or influence a specific behaviour, and educational interventions, in which information is given to a patient to increase knowledge; or both. ${ }^{7}$ It is thought that patients who are educated to understand their condition and the treatment will be more informed and have more control over their own care. These sets of patients are more likely to comply with their prescribed medication. ${ }^{8}$ Educational interventions for improving adherence aim at instructing and motivating patients about their condition and treatment through one-on-one verbal counseling, written materials and videos. ${ }^{7}$ Several studies have been conducted to test the effect of verbal and written counselling given to patients. ${ }^{9}$ Conclusions drawn from a Cochrane review found that the combination of both verbal and written health information improves patients' knowledge and satisfaction. ${ }^{9}$ Thus, patient education seems a reasonable way for shaping adherence to medicines. ${ }^{10}$

Community pharmacists play a key role in supporting patients' selfcare and medication counselling, as they are more easily accessible in the community than other HCPs. ${ }^{11}$ Furthermore, community pharmacists offer two advanced services to support patients' self-care; these are Medicines Use Reviews (MUR) and New Medicines Service (NMS). ${ }^{11}$

Omran et al. ${ }^{12}$ carried out a study to review articles that were published on pharmacist's intervention to improve medication adherence. Following a detailed research, eight most suitable articles were chosen and explored. Of the eight articles, five included views of community pharmacists and three included hospital based pharmacists. Different methods of improving adherence were used in the eight studies, some of which were, education based that include, telephone follow up, handing out leaflets and face to face sessions. 
Five studies showed that adherence rates were higher in patients receiving some sort of intervention. ${ }^{12}$ However, a study by Geffen et al. ${ }^{13}$ examining patients satisfaction on cardiovascular medications received from pharmacies, reported that $58 \%$ were unsatisfied with the information they received.

Borgsteede et al. ${ }^{4}$ investigated patients' informational needs about the medication provided to them at discharge. The study showed that patients appreciate medication counselling and preferred a combination of written and oral instructions. ${ }^{14}$ It was reported that patients regarded basic information such as the name of drugs, duration of therapy, dosage regimen and underlying conditions as important. ${ }^{14}$ Most patients also wanted to be told about the "goal" of their pharmacotherapy and possible alternatives to their current medication..$^{14}$ In accordance with other studies in this area, the results regarding need for side-effects were conflicting. Borgsteede et al. ${ }^{14}$ found that many patients preferred to receive as much side-effect information as possible. However, some patients did not wish to receive side-effect information as they were concerned that it may impact negatively on their adherence to medication. Furthermore, Borgsteede et al. ${ }^{14}$ found that most patients preferred to receive both verbal and written information. Pharmacists regularly offer verbal counseling on new prescription medicines, but written medicine information is infrequently provided. Research has shown that $40-80 \%$ of verbal medical information provided by HCPs is forgotten immediately and almost half of the information retained is remembered incorrectly. ${ }^{15}$ It is a legal requirement to supply a patient information leaflet (PIL) with each prescribed medicine. However provision of PILs does not always meet patients' needs. Often they present difficulties to patients with low literacy or those with language barrier, and the content is perceived by the reader as too technical or complex. ${ }^{16}$

Many studies have explored information needs at discharge and within the hospital setting, but few studies have explored patient medication counseling and level of satisfaction regarding counseling received from community pharmacists. This study, therefore, aimed to explore the views of patients with LTC about medication counseling from community pharmacies and the role of the PIL with this regard. The focus on patients with LTC was due to their long term use of medication and the known statistics about their low level of adherence with medication and its consequences on patients' outcomes. Knowledge obtained from this study will be helpful in improving the role of community pharmacists in patient's education to support medication adherence and will thus enhance patients' satisfaction.

\section{Methods}

A cross sectional survey of patients was undertaken. A structured questionnaire was created exploring the rate of counseling, content and format of medication counseling, satisfaction of counseling, use of the PIL and importance of the PIL. The questionnaire consisted of closed ended questions (Table 1) (Table 2). The multiple responses given were chosen following consultation of the following documents; the National Institute for Health and Clinical Excellence (NICE) guidance on medication adherence, ${ }^{17}$ the Royal Pharmaceutical Society quick reference guide on Counselling patients on medicines ${ }^{18}$ and the Medicines and Healthcare Products Regulatory Agency best practice guidance on PILs. ${ }^{19}$ A 5-point Likert scale was used to assess patient level of satisfaction in medication counseling, the volume of information included in PILs and its usefulness (Table 1) (Table 2). A pilot study was run involving 24 patients selected at random to ensure face and content validity prior to the start of the main study. The validation showed that patients were not receiving counselling by community pharmacists; hence we added the questions on NMS and MUR (Table 1), to explore other forums where patients may receive medication education. With regards to face validity, the responders suggested using bold to highlight key words in the stem of questions. Patients ( $\mathrm{N}=486)$ were recruited from community pharmacies and in public shopping centres in various areas in South and North London. The inclusion criteria were as follows; patients who suffer from a chronic condition, who are on regular medication and who collect their prescription from community pharmacies. Patients included need to be over 18years old and able to consent to the study. The study was undertaken in the period from August 2013-February 2014. Questionnaires were completed face-to face by the patient or the researcher based on patient preference. The survey was ethically approved by the Faculty of Science, Engineering and Computing ethics committee at Kingston University. Data collected from the selfcompletion questionnaires were entered and analysed using Excel. Descriptive statistics were used to analyze the closed questions of the perception questionnaires (Table 1) (Table 2).

\section{Results}

\section{Rate of medication counselling}

The population surveyed was nearly split between females (48\%) and males $(52 \%)$. The age range was from greater than 18 to greater than 65 years old with a modal age range of 35-45.

Results from this study demonstrated that only $48 \%$ ( $n=233 / 486$ ) of patients were counselled on their medication. Majority of patients who received medication counselling were provided this through the MUR or NMS services (Table 3).

\section{Content of counselling provided}

When the content of medication counselling was explored, we found that the patients commonly received generic information such as, how and when to take the medicine $(99 \%, n=231 / 233)$ and what the medicine is used to treat $(97 \%, \mathrm{n}=226 / 233)$ and duration of therapy $(80 \%, \mathrm{n}=186 / 233)$. However, only $50 \%(\mathrm{n}=117 / 233)$ patients received information about side effects with $14 \%(\mathrm{n}=33 / 233)$ being informed about the action to take following a major side-effect. With regards to lifestyle advice, information with this regard was only provided to $29 \%(n=68 / 233)$ of the patients (Figure 1$)$.

When patients were asked what extra information they wanted to receive from pharmacy medication counselling. The majority $83 \%(n=193 / 233)$ wanted extra information on the side effects of medicines, $58 \%(135 / 233)$ wanted information on where to refer to for more information and $48 \%(112 / 233)$ wanted more information on lifestyle/dietary advice.

\section{Format of counselling delivery}

When patients were questioned about the format in which the medication counselling was delivered to them, it was seen that $80 \%(n=186 / 233)$ of the patients were given counselling only verbally with only $1 \%(n=3 / 233)$ patients reporting to have received written information. In addition, it was seen that $19 \%$ patients $(n=44 / 233)$ from the total number of patients who were counselled received both verbal and written information about their medication. The written information in 54\% of the patient was provided via the PIL and for the 
remainder using other booklets/pamphlets. Majority of the patients preferred pharmacists to use both verbal and written method $(80 \%$, $\mathrm{n}=389 / 486$ ) of medication counselling.

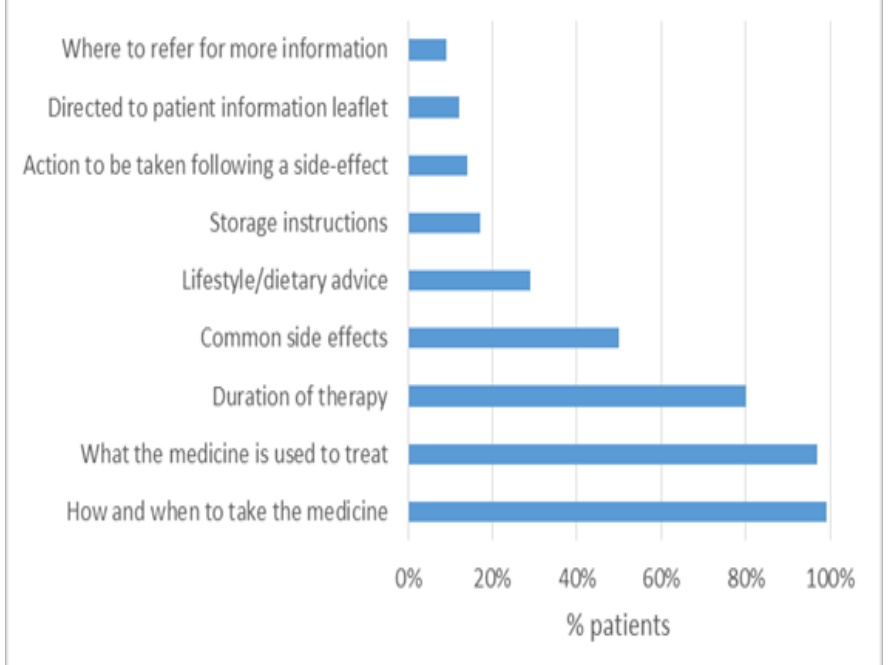

Figure I Information included in counselling received.

\section{Use of the PIL}

More than half of the participating patients read the PIL $(60 \%$, $n=292 / 486), 27 \%(n=131 / 486)$ of patients do not read the PIL, and $13 \%(n=68 / 486)$ of patients are not aware of the PIL. The main reason given by most patients (77\%) for not reading the PIL is previous counselling by an HCP. More than half of the patients (58\%) who read the PIL indicated that it contains the right amount of information and that its use for drug information is useful (51\%). As per the responses received, the main use of the PIL by patients is for the following information: the side effects of the drug $(78 \%)$, the purpose of using the drug (50\%), and how to take the drug (31\%).

\section{Satisfaction with counselling}

On a scale of 1 to 5 with 1 not being at all satisfied and 5 extremely satisfied, the majority of all patients rated their satisfaction as $3(47 \%)$ or $4(34 \%)$, showing patients either had average satisfaction or were satisfied with this information provided. Patients who rated their satisfaction as average or below average (2) (19\%) were questioned about their views. Most responses (55\%) indicated 'not enough information provided during mediation counselling' as the most common reason. The next most common reason (33\%) was 'not enough time spent'. Language barrier, lack of resources used and could not recall information were other reasons given.

\section{Discussion}

Medication counselling is one of the key interventions to enhance patients' knowledge and understanding of their medications and how and why to take them, hence enhancing adherence. Community pharmacists can play a key role in this regard. Our results show that only $48 \%$ of the patients with LTC who receive their prescribed medications from community pharmacies are being counselled. This could be due to the fact that pharmacists do not recognise patient counselling as their responsibility. Auyeung et al. ${ }^{20}$ states that pharmacists were traditionally not involved in patient education and therefore physicians and nurses feel that informing patients about primary information such as action and purpose of the medicine is their responsibility alone. Another potential reason is language barrier due to the large ethnic minority population in London.

What was interesting is that the majority of patients $(63 \%)$ who were counselled received their counselling via either the MUR or NMS service. Thus, these services are being recognised by patients as a forum for receiving medication counselling. Nevertheless, patients showed average satisfaction with counselling received mainly due to not enough information being provided and counselling being rushed.

Studies have shown that patients require detailed information about their medication including side effects. The results indicate though that the counselling received by patients included mainly generic information about a medicine i.e. purpose and dosing regimen, but lacked detailed information on side effects, what to do when suffering a side effect and lifestyle and dietary advice. This agrees with Kerzmanet al. ${ }^{21}$ who demonstrated in their study that there was a prominent existing gap of knowledge on lifestyle changes and side effects in spite of most patients being aware about the purpose of their medication. It is thus not surprising that patients, in our study, wanted more information regarding those two elements in addition to sources to refer to for more information. Ziegler et al. ${ }^{22}$ investigated the amount of information that patients want regarding their medication from physicians. From 2500 participants questioned, $76.2 \%$ wanted to be informed of all side effects associated with the medication despite how rare or common they may be. A greater number of patients $(83.1 \%)$ also desired to be informed of any serious adverse effect no matter how rare they are.

Hamrosiet al. ${ }^{23}$ reported that patients want written information, however they are generally not supplied with it. Time constraints, possible creation of patient anxiety, low literacy, and perceived length and complexity of the information were common reasons for not providing it.

Borgsteede et al..$^{14}$ have also emphasised the need for provision of written information to patients. A recent study conducted by Bjerrum et al. ${ }^{24}$ emphasised patients tend to forget or ignore nearly half of what they are told by their HCP, often leading to noncompliance. Our results however show that $80 \%$ of patients only received verbal counselling with only $20 \%$ indicating that they received written counselling. This indicates that patients do not recognize the PIL provided with each dispensed medicine as a source of written counselling information. This agrees with our previous findings from a study conducted in secondary care, where patients did not recognize the PIL as a source of written counselling information. ${ }^{25}$ This could be due to the fact that pharmacists do not highlight this as a source of information as only $12 \%$ of patients indicated that they were directed to the PIL as a source of information by the pharmacist.

When patients were questioned about the PIL, more than half of the patients were found to read the PIL with the main reason for reading it being to acquire more information about side effects. Raynor $^{26}$ found that majority (97\%) of the patients read section 'side effects' further highlighting the importance of this section for patients. Patients who read the PIL found it useful and contain the right amount of information. This finding is supported by a study conducted by Krass et al., ${ }^{27}$ where more than half of the respondents said that the quantity of information in each PIL section is just the right amount.

It must be noted that effective counselling by an HCP was found 
to be a deterrent from reading the PIL. This indicates that the PIL needs to be highlighted more as a source of medical information when counselling patients, as patients who use it find it useful. Dyck et al. ${ }^{28}$ reported in their study that the medication leaflet was referred to in only $50 \%$ of the patient counselling sessions, where pharmacists underlined or highlighted parts of the text to their patients. However, our study shows that patients don't recognize the PIL as written counselling information, maybe due to the latter not being emphasised by the HCP.

Considering that a third to half of patients with LTC do not adhere to their medication, it is essential that measures are taken in community pharmacy to enhance the level and quality of medication counselling with the PIL being used as a tool to provide detailed written medication information. Based on the sample population surveyed, it is clear that there is a mismatch between patients' information needs and what is being provided. It is thus essential that a follow up study is conducted to understand community pharmacists' views on the topic, the tools and format used for counselling and the barriers for delivery.

\section{Acknowledgements}

None.

\section{Conflict of interest}

Author declares that there is no conflict of interest.

\section{References}

1. Department of Health. Improving chronic disease management

2. Department of Health. Chronic disease management and self-care.

3. Royal College of National Practitioners. Medication Adherence: involving patients in decisions about prescribed medicines and supporting adherence.

4. Haynes RB, Ackloo E, Sahota N, et al. Interventions for enhancing medication adherence. Cochrane Database Syst Rev. 2008:(CD000011).

5. Martin LR, Williams SL, Haskard KB, et al. The challenge of patient adherence. Ther Clin Risk Manag. 2005;1(3):189-199.

6. DeFulio A, Silverman K. The use of incentives to reinforce medication adherence. Prev Med. 2012;55(Suppl):S86-S94.

7. Touchette DR, Shapiro NL. Medication Compliance, Adherence and Persistence: Current Status of Behavioural and Educational Interventions to Improve Outcomes. JMCP. 2008;14(6):S2-S10.

8. Department of Health. Liberating the NHS: No decision about me, without me.

9. Johnson A, Sandford J. Written and verbal information versus verbal information only for patients being discharged from acute hospital settings to home. The Cochrane collaboration. 2008;20(4):423-429.

10. Fang MC, Machtinger EL, Wang F, et al. Health literacy and anticoagulation-related outcomes among patients taking warfarin. Journal of General Internal Medicine. 2006;21(8):841-846.
11. Edwards A, Elwyn G. Shared decision making in healthcare: Achieving evidence-based patient choice.

12. Omran D, Guirguis LM, Simpson SH. Systematic Review of Pharmacist Interventions to Improve Adherence to Oral Antidiabetic Medications in People with Type 2 Diabetes. Canadian Journal of Diabetes. 2012;36(5):292-299.

13. Geffen ECV, Philbert D, Boheemen C, et al. Patients' satisfaction with information and experiences with counseling on cardiovascular medication received at the pharmacy. Patient Educ Couns. 2011;83(3):303-309.

14. Borgsteede SD, Karapinar-Carkit F, Hoffmann E, et al. Information needs about medication according to patients discharged from a general hospital. Patient Educ Couns. 2011;83(1):22-28.

15. Margolis, Robert H. what do your patients remember? The Hearing Journal. 2004;57(6):10,12,16-17.

16. Dyck A, Deschamps M, Taylor J. Pharmacists' discussion of medication side effects: a descriptive study. Patient EducCouns. 2005;56(1):21-27.

17. National Institute for Health and Care Excellence. Medicines Adherence: Full Guideline.

18. Counselling patients on medicines: A quick reference guide. Royal Pharmaceutical Society.

19. Medicines and Healthcare Products Regulatory Agency best practice guidance on patient information leaflets.

20. Auyeung V, Patel G, McRobbie D, et al. Information about medicine to cardiac in-patients: Patient satisfaction alongside the role perceptions and practices of doctors, nurses and pharmacists. Patient Educ Couns. 2011;83(3):360-366.

21. Kerzman H, Baron-Epel O, Toren O. What do discharged patients know about their medication? Patient Educ Couns. 2005;56(3):276-282.

22. Ziegler DK, Mosier MC, Buenaver M, et al. How much information about adverse effects of medication do patients want from physicians? Arch Intern Med. 2001;161(5):706-713.

23. Hamrosi KK, Raynor DK, Aslani P. Pharmacist and general practitioner ambivalence about providing written medicine information to patients-A qualitative study. Res Social Adm Pharm. 2013;9(5):517-530.

24. Bjerrum L, Foged A. Patient information leaflets-helpful guidance or a source of confusion? Pharmacoepidemiol Drug Saf. 2003;12(1):55-59

25. Kayyali R, Naik M, Ugbekile I, et al. Investigation of adherence measures provided to patients with newly prescribed medications prior to their discharge from secondary care. Int $J$ Pharm Practice. 2012;20(2):23-24

26. Raynor DK, Silcock J, Knapp PR, et al. How do patients use medicine information leaflets in the UK? Int J of Pharm Prac. 2007;15(3):209-218.

27. Krass I, Svarstad BL, Bultman D. Using alternative methodologies for evaluating patient medication leaflets. Patient Educ Couns. 2002;47(1):29-35.

28. Dyck A, Deschamps M, Taylor J. Pharmacists' discussion of medication side effects: a descriptive study. Patient Educ Couns. 2005;56(1):21-27. 